\title{
CONDICIONES PSICOSOCIALES Y DE SALUD GENERAL EN UNA MUESTRA DE CONDUCTORES DE BUSES DE TRANSPORTE PÚBLICO DE PASAJEROS.
}

GENERAL HEALTH AND PSYCHOSOCIAL CONDITIONS IN A SAMPLE OF PUBLIC TRANSPORT BUS DRIVERS.

CONDIÇÕES PSICOSSOCIAIS E DE SAÚDE GERAL EM UMA AMOSTRA DE MOTORISTAS DE ÔNIBUS DO TRANSPORTES PÚBLICO DE PASSAGEIROS

Leonardo Aguirre Aranibar $^{1}$

${ }^{1}$ Leonardo Aguirre es Psicólogo y realiza un doctorado en Psicología Clínica y de la Salud en la Universidad Autónoma de Madrid. Actualmente es Jefe de Proyectos de Investigación Aplicada en la Gerencia de Gestión del Conocimiento de Mutual de Seguridad C.Ch.C.

Este artículo surge de una investigación mayor financiada por Mutual de Seguridad C.Ch.C.

Trabajo recibido: 07 de diciembre 2016.

Aprobado: 01 de marzo

\section{Resumen}

Se realizó un estudio de corte transversal analítico en una muestra de 302 conductores de una empresa de transporte de pasajeros para describir cuáles son la características psicosociales y de salud general de conductores de buses del transporte público de pasajeros de Santiago de Chile. El 98.3\% fueron hombres con un promedio de edad de 48,6 años, una media de peso de $84 \mathrm{kgs}$ y una media de IMC de 28.9. El 77\% de estos indicaron que no hace ningún tipo de ejercicio y según el test de riesgo psicosocial, en 4 de las 5 dimensiones más del $50 \%$ de los trabajadores fue está en alto riesgo. Dada la poca actividad física que realizan y a los riesgos psicosociales a los que están sometidos, existe un alto riesgos de que se puedan generar patologías que afecten gravemente la salud de estos.

Palabras clave: Riesgo psicosocial, conductores, salud general. 
After signing informed consent, an analytical cross sectional study was carried out in a sample of 302 drivers from a company of passengers transport in Santiago, Chile in order to describe psychosocial and general health characteristics of bus drivers in the city. Ninety eight point three percent were men with average age of 48.6 years old, weight mean $84 \mathrm{kgs}$ and BMI mean of 28.9. From them, 77\% said they do not do any type of exercise and according to the psychosocial risk test, more than $50 \%$ of the workers is at high risk in 4 out of 5 dimensions. Given the little physical activity they do and the high psychosocial risks they are subject to, there is high risk to develop pathologies that could have a great impact on the drivers' health.

Key Works: Psychosocial risk, drivers, general health

\section{Resumo:}

Na tentativa de descrever quais são as características psicossociais de saúde em geral de motoristas de ônibus do transporte público de passageiros em Santiago (Chile), realizou-se um estudo de seção transversal analítico em uma amostra de 302 motoristas de um empresa de transporte de passageiros, após a assinatura do consentimento informado. 98,3\% eram homens com uma idade média de 48,6 anos, um peso médio de $84 \mathrm{~kg}$ e um IMC médio de $28,9.77 \%$ deles disseram que não faziam qualquer atividade física de acordo com o teste de risco psicossocial em 4 das 5 dimensões mais de $50 \%$ dos trabalhadores estão em alto risco. Por causa da baixa atividade física e dos riscos psicossociais a que estão sujeitos, há um alto risco de que possam ser geradas doenças que afetam seriamente a saúde deles.

Palavras-chave: riscos psicossociais, saúde ocupacional, motoristas de ônibus.

\section{Introducción.}

La salud de los conductores profesionales es un tema estudiado internacionalmente. No fue hasta la mitad del siglo XX que empezó la investigación en salud ocupacional de conductores de transporte urbano, en ese sentido los trabajos publicados por Morris y colaboradores (1) establecieron la naturaleza nociva potencial de la conducción profesional. Entre las variables que más se afectan la labor de conducción se pueden nombrar el medio ambiente y el estado de salud de los conductores (2). Consistentemente con eso, según la Administración de la Seguridad del Tráfico en Autopistas (NHTSA, por sus siglas en inglés), el factor humano estaría implicado entre el $71 \%$ al $93 \%$ de los accidentes de tráfico; los factores de la vía, entre el 12\% y el $34 \%$; y los del vehículo, entre el 4,5\% y el $13 \%$. (3)

Por otro lado, los factores de salud que afectan la capacidad para conducir se encuentran las enfermedades que pueden perturbar la conciencia, como es el caso del infarto del miocardio, la hipertensión arterial, enfermedades cerebrovasculares, la isquemia cerebral, crisis vasovagal, epilepsia e hipoglicemia, entre otras. También son conocidas las influencias transitorias que provocan el alcohol, determinados medicamentos, las drogas y la fatiga. (2).

Las vibraciones a las que están sometidos los conductores, la posición sedentaria en la conducción, los cambios de turnos de manera irregular entre otros, hacen que los conductores estén más susceptibles a sufrir trastornos de tipo musculo esqueléticos. (4). Además, los conductores profesionales suelen estar expuestos a lesiones y/o muertes a causa de accidentes de tránsito. Ellas pueden provocar trastornos por estrés postraumático la que, de no ser tratada oportunamente, no solo pueden generar problemas físicos sino 
que también burnout y abusos de sustancias. $(5,6,7)$

Los conductores también están sometidos a un nivel alto de estrés laboral, más que trabajadores de otras ocupaciones. $(8,9,10,11,12)$. La conducción de transporte público urbano constituye uno de los oficios más estresantes (13) y que está íntimamente vinculada al manejo de vehículos: el estrés modula en buena medida la forma de conducir de las personas y puede ser la causa de un número considerable de accidentes y además puede ocasionar efectos negativos en el conductor como generación de mayores niveles de hostilidad y de comportamientos competitivos; mayor tendencia a la impaciencia, toma de decisiones arriesgadas y conducción imprudente; disminución de la concentración; y el efecto negativo que tiene el uso incontrolado de fármacos, alcohol u otras sustancias que se pueden utilizar para reducir el estrés (13).

Se han identificado aspectos que pueden mejorar las condiciones de salud en el trabajo en pos de disminuir las situaciones estresantes. El dar mayor control a los conductores es una buena medida para disminuir la sintomatología estresante (14) lo que permitiría, entre otras cosas, prevenir la fatiga que sufren los conductores, en especial, los que laboran en sistema de turnos (15)

La realidad Chilena no es ajena a estas condiciones y se ha intentado estudiar como los sistemas de transporte afectan la salud y calidad de vida de las personas. En Chile, el nuevo sistema de transporte de la ciudad de Santiago, llamado "Transantiago" fue pensado para cambiar la organización del transporte colectivo existente en la urbe. Este sistema integrado de buses y tren subterráneo que opera en la capital de Chile desde 2007, cuenta con un total de 371 servicios de superficie que recorren 34 comunas del área metropolitana y cuya operación es responsabilidad de siete empresas privadas, concesionarias de los recorridos a través de contratos de prestación de servicios con el estado chileno. La red alcanza un total de 11.395 kilómetros de servicios, con más de 4 millones de viajes. El Transantiago reformó por completo la malla de recorridos de las antiguas "micros" (buses urbanos), diseñando un sistema basado en el uso de servicios alimentadores y troncales, en conjunto con el Metro de Santiago.

Una sola empresa de este sistema de transporte puede realizar más de siete mil expediciones diarias, movilizar alrededor de 500 mil personas cada día jornada. Los sistemas de jornadas laboral en la que operan los conductores son diversos aunque el más frecuente es el de tipo "Jornada Excepcional de Trabajo" con 9 horas de trabajo para un día laboral normal de lunes a viernes y de 10 horas diarias en sábados, domingos y festivos, considerando un tiempo de colación de 45 y 40 minutos.

Este sistema de jornada excepcional y las condiciones de trabajo a la que están expuestos los conductores de buses y los efectos de estas en la salud de los conductores, no ha sido aún estudiada con profundidad. Por ello la finalidad de este estudio es describir las condiciones de salud y los principales riesgos psicosociales a los que están expuestos los conductores de vehículos de transporte público de pasajeros.

\section{Material y métodos}

Objetivo General: Describir las condiciones psicosociales y de salud general en conductores de buses de una empresa de transporte público de pasajeros en la ciudad de Santiago de Chile.

\section{Método}

Diseño: El presente estudio corresponde a un diseño de corte transversal analítico. Muestra

La población correspondió a conductores de buses de una empresa de transporte público de pasajeros de la ciudad de Santiago de Chile, cuya población era de 1667 conductores. 
La muestra se obtuvo de manera probabilística mediante un muestreo aleatorio simple de la planilla de conductores de dicha empresa. Para el cálculo del tamaño muestral se utilizó un $5 \%$ de margen de error y un $95 \%$ de confianza, asumiendo heterogeneidad poblacional máxima (50\%), la muestra estuvo compuesta por 310 conductores de buses.

Para la selección de los conductores se utilizaron los siguientes criterios de inclusión: (a) que los trabajadores realizaran jornada completa en el sistema de jornada excepcional y (b) que poseyeran una antigüedad igual o mayor a seis meses en la empresa.

Instrumentos: En este estudio se aplicaron 3 instrumentos psicométricos: El test Nasa TLX, el test SUSESO-Istas21 versión corta y una encuesta de salud general. El Nasa TLX es un test que mide el grado en que un trabajador se encuentra respecto a su estado de atención y de concentración el que, cuando se realiza conscientemente y con cierta continuidad, da lugar a la carga mental. El NASA-TLX fue desarrollado por el Aerospace Human Factors Research Division de la NASA, en su centro de investigación de Ames (16). Es un procedimiento de valoración multidimensional que entrega una puntación global de carga de trabajo, basada en una media ponderada de las seis subescalas. El NASA-TLX ha mostrado valores de consistencia interna moderados de 0,69 medidos con el Alpha de Cronbach(17)

Este instrumento no posee validez nacional pero es la herramienta que se ha definido como la técnica a utilizar para evaluar la carga mental, por parte de la Superintendencia de Pensiones en su "Guía Técnica para la Evaluación de trabajo Pesado" (18)

E1 SUSESO-Istas21es un instrumento de evaluación de riesgos psicosociales que permite identificar y medir aquellos factores derivados de la organización del trabajo que constituyen riesgo para la salud. Es la versión chilena de la versión española del instrumento original COPSOQ (19). La versión aplicada en este estudio fue la versión corta que consta de 20 ítems con formato de respuesta tipo Likert, que posee 5 alternativas de respuesta desde "Siempre" hasta "Nunca". Consta de 5 dimensiones que evalúan diversos componentes del riesgo psicosocial: Exigencias psicológicas en el trabajo, Trabajo activo y desarrollo de habilidades, apoyo social en la empresa y calidad del liderazgo, compensaciones y doble presencia. El SUSESO-Istas 21 ha sido validado en la población chilena por Alvarado et.al (20).Sus valores de consistencia interna para cada dimensión varían entre 0,64 y 0,92, medidos con el Alpha de Cronbach.

Por último, la encuesta de salud general aplicada en este estudio se construyó en base a las preguntas de la Encuesta Nacional de Empleo, Trabajo, Salud y Calidad de Vida de Trabajadores y Trabajadoras en Chile (ENTS). La ENTS es una encuesta que se realiza cada dos años y surge de la alianza entre el Ministerio de Salud, la Dirección del Trabajo y el Instituto de Seguridad Laboral (21). La encuesta aplicada en este estudio se basa en el módulo de Calidad de Vida y Salud. Dicho módulo consta de 67 preguntas que apuntan diversas áreas de la salud de los trabajadores, desde enfermedades físicas, psicológicas, sueño, estado de salud general, etc. De la totalidad de preguntas, fueron seleccionadas 14, en base a la pertinencia de los aspectos a evaluar en los conductores.

Procedimientos:La aplicación de los instrumentos fue realizado en coordinación con el departamento de prevención de riesgos de la empresa participante cuidando que la aplicación de los test se realizara durante los cursos de capacitación que los conductores. Se les solicitó un tiempo para que pudieran participar del proceso de investigación y a aquellos que deseaban participar, se les pidió confirmar mediante la firma de un consentimiento informado.

Análisis estadísticos: Se realizó un análisis cuantitativo de la información recolectada por los test, mediante estadística descriptiva y pruebas de correlaciones para determinar asociación entre diferencias variables. Las variables continuas se describen con su media aritmética y las variables discretas, mediante proporciones. Para las pruebas de asociación 
para variables discretas, se utilizó la prueba de Chi-Cuadrado para dos muestras y la Correlación de Spearman(rho) para establecer la fuerza de la asociación. Se utilizó el programa estadístico SPSS v.23. En todas las pruebas de asociación se utilizó un valor de significación del 0,05

Aspectos Éticos: El proyecto fue presentado a evaluación y aprobado por el Comité de Ética Científico de Mutual de Seguridad de la Cámara Chilena de la Construcción (CChC). Para la aplicación de los test se solicitó a los conductores de buses su participación libre y voluntaria mediante la firma del consentimiento informado. El análisis de la información se realizó de manera anónima mediante la desvinculación los datos de identificación (nombre, rut, etc.) de las respuestas entregadas a los test por cada conductor de tal manera de que esta información fue analizada y evaluada en ciego.

\section{Resultados}

La muestra estimada inicialmente fue de 310 conductores aunque al finalizar la etapa de aplicación general se pudo medir a 302, de los cuales 297 (96,4\%) eran hombres y 5 mujeres $(1,7 \%)$, con un promedio de edad de 46 años (DS=9,1), un peso de $84 \mathrm{Kg}(\mathrm{DS}=15,2)$ y un Índice de Masa Corporal (IMC) promedio de 28.9 ( $\mathrm{DS}=4,54)$. Si bien es cierto, no se alcanza es exactamente el tamaño muestral estimado (la diferencia es de 8 conductores menos), la diferencia no es estadísticamente significativa y no tiene efecto en la disminución de la potencia estadística de las pruebas de análisis a utilizar.

Respecto a la evaluación de la carga mental, y siguiendo al Decreto Supremo No 71, existen dos maneras de presentar los resultados de este test. La primera de ellas es indicando la puntuación promedio obtenida. Es así como se obtuvo un puntaje global de 1242 puntos el que se ubica en un rango alto de carga mental. Los aspectos de su trabajo mantienen a los conductores en un nivel alto de atención y concentración mental: ello que implica que las mayores dificultades en el trabajo se deben más a factores organizacionales fácilmente modificables, más que a cuestiones estructurales.

Ahora bien, y siguiendo la segunda forma de interpretar los datos del NASA TLX, es necesario identificar cual o cuales son las dimensiones que para los conductores son las que más efecto tienen sobre la carga mental general. En ese sentido, los conductores indicaron que, de mayor puntaje a menor puntaje ponderado, las dimensiones que más afectan la carga mental son:

Tabla $N^{\circ} 1$ Puntuaciones Ponderadas para cada Dimensión. Test Carga Mental Nasa TLX

\begin{tabular}{|lc|}
\multicolumn{1}{|c}{ Dimensión } & Puntuación Ponderada \\
\hline Exigencias Mentales & 323 \\
\hline Esfuerzo & 262 \\
\hline Rendimiento & 253 \\
\hline Frustración & 233 \\
\hline Exigencias Temporales & 231 \\
\hline Exigencias Físicas & 193 \\
\hline
\end{tabular}

Según la información de la Tabla $\mathrm{N}^{\circ} 1$, se puede observar que tanto las exigencias mentales como el esfuerzo son las dos dimensiones que poseen mayor puntaje y, por ende, son las 
que más tienen injerencia en la carga mental general. En contraposición, los conductores evalúan que las exigencias físicas no son tan relevantes y no se resienten tanto, lo cual es consistente con su labor dado que esta no demanda mayores esfuerzos físicos o musculares.

Respecto a la evaluación de riesgo psicosocial, el SUSESO - Istas21no es un test que de un puntaje general de riesgo psicosocial (como el NASA TLX) sino que, al aplicarlo masivamente, nos permite identificar cuantos trabajadores están expuestos a distintos niveles de riesgo psicosocial. .

Es así como se puede observar en él gráfico $\mathrm{N}^{\circ} 1$ que en todas las dimensiones, menos la dimensión de doble presencia, más del $50 \%$ de los conductores están en alto riesgo psicosocial. La dimensión (en orden descendiente) en la que más conductores están en riesgo es la de apoyo social, seguido por exigencia psicológica, compensaciones, trabajo activo, terminando con doble presencia.

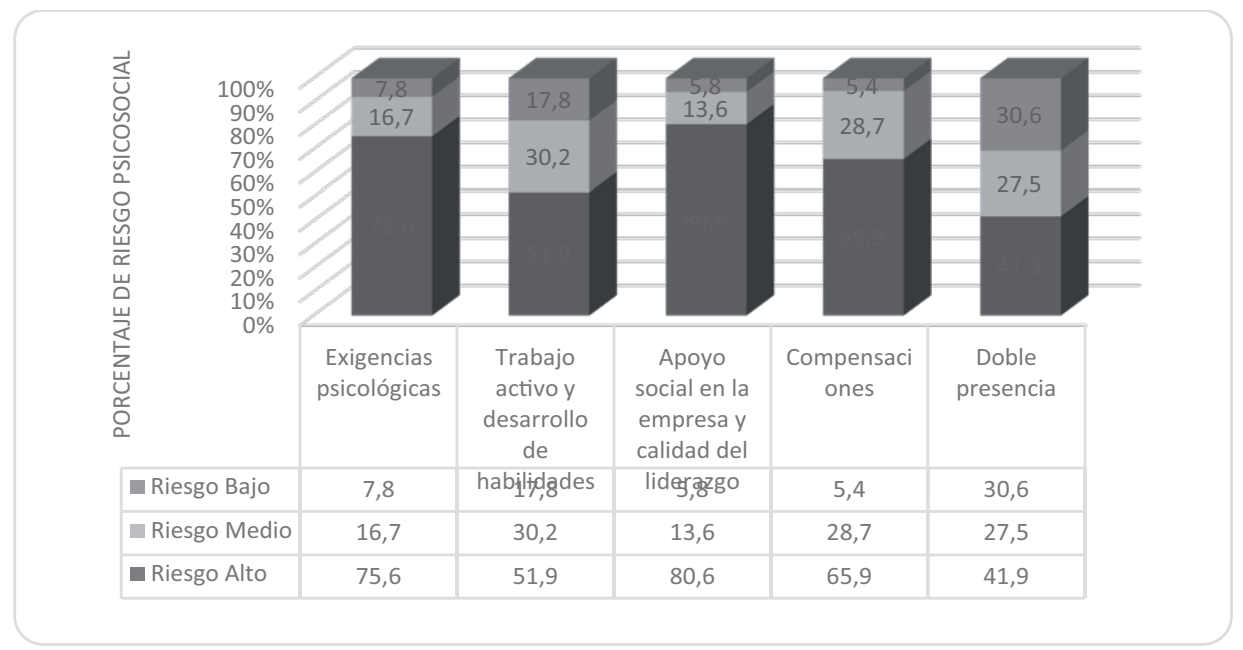

Gráfico $\mathbf{N}^{\circ} \mathbf{1}$ Resultados del SUSESO-Istas21

El gráfico $\mathrm{N}^{\circ} 1$ nos indica que los conductores están sometidos a un alto nivel de riesgo psicosocial en la mayoría de las dimensiones que evalúa este test.

Respecto a los resultados de la encuesta de salud general, se observó que existen diversos problemas de salud prevalentes en la muestra estudiada. Así, por ejemplo, el 46\% de los conductores indica que su salud general es peor que hace un año; el 60\% realizan poca actividad física, generalmente solo una vez al mes.

Respecto a la alimentación, la mayoría sí toma desayuno (76\%), se alimenta en el horario de trabajo (43\%) dado que poseen las condiciones físicas adecuadas para hacerlo.

Cuando se les pregunta respecto a enfermedades y sintomatología dolorosa, el $71 \%$ menciona que en los últimos 12 meses ha sufrido dolores musculares, siendo los dolores de columna y cuello, los más prevalentes (63\%).

El consumo de alcohol y cigarros está controlado, según lo informado por ellos mismos ( $83 \%$ bebe menos de una vez al mes y $57 \%$ fuma), aunque estos valores están claramente mediados por la deseabilidad social. Sin embargo, lo que más preocupa es que el $83 \%$ menciona que su calidad de sueño es mala o regular.

Si relacionamos los niveles de riesgo psicosocial con los aspectos de salud general se puede observar que las personas que poseen un riesgo alto de exigencia psicológica evalúan su salud en un nivel peor que hace un año $(X 2=32,95 ; \mathrm{gl}=8 ; \mathrm{p}=0,00)$, con poca frecuencia toman desayuno $(\mathrm{X} 2=11,12 ; \mathrm{gl}=6 ; \mathrm{p}=0,032)$, no tienen tiempo para almorzar $(\mathrm{X} 2=18,71$; 
$\mathrm{gl}=2 ; \mathrm{p}=0,00)$, no cuentan con un lugar para almorzar $(\mathrm{X} 2=10,48 ; \mathrm{gl}=2 ; \mathrm{p}=0,005)$, han tenido dolores musculares en los últimos 12 meses $(\mathrm{X} 2=19,13 ; \mathrm{gl}=2 ; \mathrm{p}=0,00)$ y tienen peor calidad de sueño $(X 2=35,32 ; \mathrm{gl}=4 ; \mathrm{p}=0,00)$.

Respecto a los conductores con alto nivel de riesgo en la dimensión Trabajo activo y desarrollo de habilidades, estos manifiestan que su salud en un nivel peor que hace un año $(\mathrm{X} 2=22,28 ; \mathrm{gl}=8 ; \mathrm{p}=0,004)$, hace poca actividad física $(\mathrm{X} 2=27,52 ; \mathrm{gl}=6 ; \mathrm{p}=0,00)$, fuman $(\mathrm{X} 2=12,65 ; \mathrm{gl}=6 ; \mathrm{p}=0,00)$ y tienen peor calidad de sueño $(\mathrm{X} 2=31,03 ; \mathrm{gl}=4 ; \mathrm{p}=0,00)$.

En relación al alto riesgo en la dimensión apoyo social en la empresa, los conductores manifiestan que su salud es peor que la de hace un año $(X 2=22,26 ; \mathrm{gl}=8 ; \mathrm{p}=0,004)$ y tienen peor calidad de sueño $(\mathrm{X} 2=22,33 ; \mathrm{gl}=4 ; \mathrm{p}=0,000)$.

Finalmente, las personas que poseen un alto riesgo psicosocial en la dimensión compensaciones realizan poco deporte $(\mathrm{X} 2=14,11 ; \mathrm{gl}=6 ; \mathrm{p}=0,028)$, no pueden tomarse tiempo para almorzar $(\mathrm{X} 2=6,28 ; \mathrm{gl}=2 ; \mathrm{p}=0,043)$, beben más alcohol $(\mathrm{X} 2=27,34$; $\mathrm{gl}=8 ; \mathrm{p}=0,001)$ y tienen peor calidad de sueño $(\mathrm{X} 2=12,51 ; \mathrm{gl}=4 ; \mathrm{p}=0,014)$

Como se puede observar, la variable que consistentemente se relaciona con el alto riesgo es la percepción de la calidad de salud general actual y la calidad del sueño. En la primera de las relaciones, a peor calidad de salud y sueño mayor es el riesgo psicosocial se relacionan positivamente con el riesgo psicosocial $(\mathrm{rho}=0,331$ para calidad de salud y rho $=0334$ para calidad de sueño).

Es así como el tiempo dedicado al descanso es bajo y la calidad del sueño se percibe como mala por parte de los conductores, lo que puede afectar aún más la salud física y psíquica. Los tiempos de traslado (trabajo-hogar-trabajo) están afectados, en general, por los tipos de turno y por la distancia que existe entre el lugar de trabajo y el hogar del trabajador. De hecho, el promedio de tiempo dedicado para el traslado hogar-trabajo-hogar es de 60 minutos en cada tramo (Rango de 10 a 180).

\section{Discusión}

Respecto a los principales resultados presentados se pueden mencionar diversos aspectos a destacar. En primer lugar hay que mencionar que el tamaño muestral real obtenido en este estudio permite hacer generalizaciones que son aplicables a todos los conductores laboran en el transporte público de pasajeros de la ciudad de Santiago. Sin embargo, la poca participación de mujeres se debe a que la proporción de estas en el marco general de los conductores es baja.

Las condiciones de carga mental, indican que los conductores poseen un nivel alto (puntaje medio global obtenido 1242 puntos), lo que en consecuencia implica que las mayores dificultades en el trabajo se deben a factores organizacionales fácilmente modificables. Este puntaje global es útil dado que refleja, respecto a la carga mental, que es posible intervenciones y modificaciones para poder disminuir los niveles de carga mental. Las modificaciones, en este aspecto, deben ir en el área organizacional del trabajo (liderazgo, sistema de turnos, entre otros.)

Si se analizan las dimensiones en función de cuál de estas posee más injerencia en la carga mental, la exigencia mental y el esfuerzo se manifiestan como las principales fuentes, consistente con los hallazgos de Hlotova, Cats\&Meijer(14). Ello se refleja en los altos niveles de atención y concentración que se debe poner en la tarea de conducción, así como en la tensión constante en la que están sometidos (esfuerzo mental). El nivel de energía que deben poner en los recorridos es tal que puede afectar su salud psicológica, elevando los niveles de estrés sin mayores instancias de compensación o donde se pueda liberar las tensiones psicológicas producidas en su trabajo. El esfuerzo físico que se pone en la tarea, no es evaluado como relevante por los conductores, lo que es consistente con la tarea de 
conducción (sedentaria). Sin embargo, la alta prevalencia de dolor de columna y cuello es consistente con lo encontrado en diversas investigaciones $(22,23)$ en donde este tipo de sintomatología dolorosa se presenta como los principales dolores musculares asociados a la tarea de conducción.

En relación al riesgo psicosocial, los conductores evalúan que en su entorno no existen las instancias para poder aliviar y/o paliar las tensiones y estresores de su trabajo. Más del $80 \%$ de los trabajadores se encuentran en alto nivel de riesgo en la dimensión apoyo social, lo que refleja que existen problemas respecto a la relación que se establece entre los conductores y las jefaturas directas, así como con la alta dirección de la empresa. El papel de la dirección y la importancia de la calidad de dirección para asegurar el crecimiento personal, la motivación y el bienestar de los trabajadores son importantes. En ese sentido, el nivel de estrés al que están sometidos es tal que se convierte en un foco de problemas psicológicos, lo cual es concordante con el test NASA TLX respecto al alto nivel de exigencia mental.

El segundo factor de riesgo psicosocial, en orden de importancia, es el de exigencias psicológicas lo que implica que los conductores evalúan que como altas las exigencias respecto a la cantidad y volumen de trabajo y el tiempo disponible para realizarlo. Además, la exigencias emocional de su trabajo (tensión al conducir, actitudes violentas del medio y de los pasajeros, etc.) provocan que en esta dimensión más del 75\% de los conductores se encuentre en riesgo.

En el otro extremo, la doble presencia (grado en que los problemas personales y de la casa afectan el trabajo) no se evalúa como fuente de riesgo psicosocial.

En lo referente a las condiciones de salud de los conductores y la poca actividad física que realizan, estas generan condiciones ideales para que se puedan producir patologías que puedan afectar la salud de los mismos. El tiempo dedicado al descanso es bajo y la calidad del sueño se percibe como mala por parte de los conductores, lo que puede afectar aún más la salud física y psíquica. Los tiempos de traslado (trabajo-hogar-trabajo) están afectados por los tipos de turno y por la distancia que en algunos casos existe entre el lugar de trabajo y el hogar del trabajador. En general los trabajadores indican que el tiempo promedio de traslado es de una hora, en rangos que van de 10 minutos hasta 3 horas. Todo ello redunda en un cansancio que se va acumulando de manera creciente.

En resumen, existen aspectos potencialmente positivos de la tarea de conducción son: (a) La carga mental está en un nivel alto aunque puede ser mejorable con modificaciones en la organización del trabajo; (b) El riesgo psicosocial se relaciona, con mayor intensidad, con la carencia percibida de apoyo por parte de la jefatura directa y de la operación, además de la tensión psicológica de la tarea de conducción; (c) La salud física de los conductores aspectos que pueden definirse como riesgosa (Peso, IMC, sueño) aunque, al igual que la carga mental, son de fácil modificación.

\section{Bibliografía}

1. Garcell, H. Estado de salud de conductores profesionales que circulan por la vía blanca. Revista Cubana de Salud Pública. 2010; Vol27, Issue 2

2. Centers for Disease Control. Alcohol involvement in fatal motor-vehicle crashes-United States, 1999-2000. MMWR - Morb Mortal Wkly Rep. 2001;50(47):1064-5

3. Chung YS, Wong JT. Developing effective professional bus driver health programs: An investigation of self-rated health. Accid Anal Prev. 2011;43(6):2093-103

4. Chen MJ, Cunradi C. Job stress, burnout and substance use among urban transit operators: The potential mediating role of coping behaviour. Work Stress [Internet]. 2008;22(4):327-40

5. Issever H, Onen L, Sabuncu HH, Altunkaynak O. Personality characteristics, 
psychological symptoms and anxiety levels of drivers in charge of urban transportation in Istanbul. Occup Med (Chic Ill). 2002;52(6):297-303

6. Vedantham K, Brunet A, Boyer R, Weiss DS, Metzler TJ, Marmar CR. Posttraumatic stress disorder, trauma exposure, and the current health of Canadian bus drivers. Can J Psychiatry. 2001;46(2):149-55

7. Kompier MAJ, Di Martino V. Review of bus drivers' occupational stress and stress prevention. Stress Med [Internet]. 1995;11(4):253-62

8. Magnusson ML, Pope MH, Wilder DG, Areskoug B. Are occupational drivers at an increased risk for developing musculoskeletal disorders? Spine (Phila Pa 1976). 1996;21(6):710-7

9. Tse JLM, Flin R, Mearns K. Facets of job effort in bus driver health: deconstructing "effort" in the effort-reward imbalance model. J Occup Health Psychol [Internet]. 2007;12(1):48-62

10. Tüchsen F, Endahl LA. Increasing inequality in ischaemic heart disease morbidity among employed men in Denmark 1981-1993: The need for a new preventive policy. Int J Epidemiol. 1999;28(4):640-4

11. Wang PD, Lin RS. Coronary heart disease risk factors in urban bus drivers. Public Health. 2001;115(4):261-4.

12. Evans GW, Carrère S. Traffic congestion, perceived control, and psychophysiological stress among urban bus drivers. J Appl Psychol. 1991;76(5):658-63

13. Isoba M. (2002) Estrés al volante. Luchemos por la vida, 20. Disponible en: http:// www.luchemos.org.ar/espa/rev20/pag14.htm.

14. Hlotova Y, Cats O, Meijer S. Measuring Bus Driver's Occupational Stress under Changing Working Conditions. Transp Res Board 93rd Annu Meet January 12-16, Washington, DC. 2014; 13-20.

15. Makowiec-Dąbrowska T, Siedlecka J, Gadzicka E, Szyjkowska A, Dania M, Bortkiewicz A, et al. Work fatigue in urban bus drivers. MedycynaPracy(2015); 66(5): 661-677.

16. Hart \&Staveland, L. S. Development of the NASA-TLX: Results of empirical and theoretical research. In: Human mental workload [Internet]. 1988. p. 139--183

17. Eva DR, Susana RV, Jesus MG, Lourdes LM. Estudio Psicométrico del Índice de Carga Mental NASA-TLX con una Muestra de Trabajadores Españoles. RevPsicol. del Trab y las Organ. 2010;26(3):191-9

18. MINTRAB (2010). Guía Técnica para la Evaluación del Trabajo Pesado. Decreto Supremo $\mathrm{N}^{\circ} 71$. Disponible en http://www.safp.cl/portal/institucional/578/ articles-8418_guia_tecnica.pdf

19. Kristensen TS, Borg V. Copenhagen Psychosocial Questionnaire. Ment Health (Lond). 2003; 5(5):5.

20. Alvarado, Ruben; Pérez, Juan, Saavedra, Nadia; Fuentealba, Claudio; Alarcón A, Marchetti, Nella; Aranda W. Validación de un cuestionario para evaluar riesgos psicosociales en el ambiente laboral en Chile. RevMedChil. 2012;140(9):1154-63

21. MINTRAB (2007) Encuesta Nacional de Condiciones de Empleo, Trabajo, Calidad de vida y Salud. Disponible en http://epi.minsal.cl/estudios-y-encuestaspoblacionales/encuestas-poblacionales/enets/

22. Szeto GPY, Lam P. Work-related musculoskeletal disorders in urban bus drivers of Hong Kong. J OccupRehabil. 2007;17(2):181-98.

23. Mozafari A, Vahedian M, Mohebi S, Najafi M. Work-related musculoskeletal disorders in truck drivers and official workers. Acta Medica Iranica. (2015, July); 53(7): 432-438. 